\title{
MEMOIRE DES BALKANS, MEMOIRES DES FRANCE(S): VERS LA RECONNAISSANCE DE MEMOIRES PLURIELLES ET NON EXCLUSIVES
}

\author{
Jean-Jacques TATIN GOURIER, Université de Tours, tatin-gourier@univ-tours.fr \\ 10.31902/fll.29.2019.3 \\ UDK 94(44:497) \\ UDK 172.15(44:497)
}

\begin{abstract}
Résumé : Le jeu contemporain de la mémoire en France et celui qui, à partir des années 1990, se développe dans les pays issus de l'ex-Yougoslavie sont de toute évidence profondément différents. Nous avons pourtant tenté une mise en parallèle en nous appuyant sur les concepts mis en place par Pierre Nora dans son ouvrage Les lieux de mémoire et plus particulièrement sur son opposition des notions de mémoire et d'histoire. En France, par le biais de l'enseignement républicain, l'histoire a longtemps imposé une tradition de mémoire nationale. Mais à partir des années 1960, la recherche historique en France a largement contribué à un détachement du traditionnel « récit national » : l'histoire s'est de plus en plus affirmée dans sa dimension de recherche sociale et anthropologique; les questions d'ordre épistémologique (l'histoire de l'histoire), théorique et méthodologique se sont de plus posées avec force. En outre les tensions mémorielles autour des événements contemporains (Seconde Guerre mondiale, décolonisations) ont retenu l'attention des chercheurs et d'un lectorat de plus en plus ample. Dans la série de brutales ruptures qui, dans les années 1990, ont vu surgir ou resurgir les différentes composantes de l'ex-Yougoslavie, la grande antithèse des espaces mémoriels (espace dominant de la Yougoslavie / espaces dominés des composantes - nationalités) s'est en quelque sorte inversée. Inégalement toutefois compte tenu des ressorts mémoriels, et par conséquent politiques, de chacune des nations nouvellement indépendantes. Des questions dès lors se posent pour chacun des Etats. Qu'est-ce qui a été effacé? Qu'est-ce qui a resurgi et à quel prix ? Quelles ont été ici et là les instrumentalisations de la mémoire.
\end{abstract}

Mots-clés : Mémoire, France, ex-Yougoslavie, Balkans, histoire, nationalisme(s), manipulation.

A partir des analyses de Pierre Nora dans son ouvrage Les Lieux de mémoire (Gallimard, 1984), nous tenterons d'envisager, dans chacun des deux principaux espaces que le colloque met en parallèle (Balkans, entendus comme ensemble des pays issus de l'ex-Yougoslavie, et France), les conditions qui ont conduit dans le passé ou conduisent présentement à des prises en compte de la mémoire, mémoire rigoureusement distinguée de l'histoire, et des lieux où elle se cristallise. Ces lieux pouvant être très divers dans chaque espace étudié : monuments avec leur symbolique, emblèmes publics et commémoratifs, toponymie des villes, rues et places, parties historiques des programmes et manuels scolaires, institutions définissant et gérant le patrimoine avec des surfaces et des géométries qui peuvent être très variables dans l'espace et le temps.

Parmi les conditions de surgissement de ce questionnement sur les lieux de mémoire et plus globalement sur la notion même de mémoire, Pierre Nora 
évoque au premier chef ce qu'il nomme « l'accélération de l'histoire ", " un basculement de plus en plus rapide dans un passé définitivement mort, la perception globale de toute chose comme disparue - une rupture d'équilibre " (Nora $1: 17)$. Les modalités de cette accélération et de ce basculement de la temporalité ont été, de toute évidence, très différentes de la France à l'espace balkanique. Dans l'éclatement de la Yougoslavie et la constitution des nouveaux états indépendants, le recours à la mémoire et son utilisation par les diverses forces en présence a joué un rôle très important comme le montre Valérie Rosoux dans son étude Pièges et ressources de la mémoire dans les Balkans (Université catholique de Louvain, novembre 2002). C'est dire que la mise en parallèle sur la question de la mémoire des Balkans et de la France ne va pas de soi. Faut-il de plus rappeler que, par-delà les diagnostics actuels sur une France " archipélisée »- profondément divisée entre une France des métropoles et des élites bénéficiant de la mondialisation et une France délaissée " des territoires ", la tradition centraliste de la France a toujours été reconduite de l'Ancien Régime à la République ? En matière de politique de la mémoire, il faut également le rappeler, la France de la Révolution de 1789-1793 a de plus été pionnière avec sa première commémoration d'état, la fête de la Fédération le 14 juillet 1790 et le développement sans précédent d'un culte des grands hommes et de rituels inédits de rappel du passé. II suffit de rappeler également la transformation de l'église Sainte Geneviève, patronne de Paris en Panthéon accueillant dès la Révolution les cendres de Voltaire et de Rousseau, des dirigeants révolutionnaires, d'ailleurs vite dépanthéonisés, Mirabeau et Marat. Une politique de la mémoire nationale reprise, amplifiée et systématisée par la Troisième République (18751940) et perpétuée jusqu'à notre époque. Non, il est vrai, sans débats et tensions qui dessinent des enjeux toujours renouvelés.

Mais il importe de revenir tout d'abord à la réflexion liminaire de Pierre Nora. Pour ce qui concerne sa distinction de la mémoire et de l'histoire, je me rapporterai plus particulièrement à son introduction personnelle du tome 1 des Lieux de mémoire, La République, ample préface intitulée « Entre mémoire et histoire. La problématique des lieux ». Dans cette introduction, Pierre Nora développe en fait trois points : "la fin de l'histoire-mémoire ", « la mémoire saisie par l'histoire ", " les lieux de mémoire, une autre histoire ". C'est autour de ces trois points que je reprendrai et commenterai dans un premier temps, que je tenterai de suggérer quelques mises en regard dans la France actuelle et dans les pays tout aussi contemporains issus de ce qui fut la Yougoslavie.

Pour ce qui est de la fin de l'histoire-mémoire, Pierre Nora fait état en incipit d'un diagnostic global qui résonne bien au-delà de la France : diagnostic du retrait voire de la disparition d'une mémoire vivante et spontanée :

"Accélération de l'histoire. Au-delà de la métaphore, il faut prendre la mesure de ce que l'expression signifie : un basculement de plus en 
plus rapide dans un passé définitivement mort, la perception globale de toute chose comme disparue - une rupture d'équilibre. [...] L'accession à la conscience de soi sous le règne du révolu, l'achèvement de quelque chose depuis toujours commencé. On ne parle tant de mémoire que parce qu'il n'y en a plus » (Nora 18).

Dès 1984, Pierre Nora souligne le fait que " le monde entier est entré dans la danse ". Et Pierre Nora d'évoquer tout à la fois l'universalité des causes profondes (mondialisation, massification, médiatisation) et l'universalité des conséquences : les sociétés nouvellement indépendantes (Pierre Nora entend par là les entités issues de la décolonisation) entraînées dans l'historicité ainsi que leurs composantes ethniques " à fort capital mémoriel et à faible capital historique ». Une décennie après la publication de l'ouvrage dirigé par Pierre Nora, avec la fin de la guerre froide et la chute des systèmes communistes, l'histoire s'est encore accélérée et a touché d'amples espaces qui pouvaient sembler jusqu'alors préservés.

Pierre Nora envisage très clairement le couple mémoire/histoire comme un couple antinomique, même si la mémoire et l'histoire ont pu se confondre pendant un temps. Il précise qu'il n'a sans doute été de mémoire " vraie, sociale » dans sa pureté que dans celle des sociétés dites archaïques où l'héritage est constamment reconduit dans les gestes, les conduites et les mots et renvoient au temps des héros, des origines et des mythes. Mais Pierre Nora évoque aussi (et nous sommes là, dans ce qu'il appelle la phase de " l'histoiremémoire ", plus proches de nous mais néanmoins déjà désuètes) des sociétés-mémoires et des idéologies-mémoires, assurant les unes et les autres, sans failles ni doutes ni interrogations, avec leurs institutions étatiques, scolaires et familiales, la conservation et la transmission des valeurs. En France l'exemple est évidemment l'histoire-mémoire de la nation telle que la Troisième République l'a massivement enseignée sans le moindre doute ni le moindre questionnement. Mais l'histoire proprement dite (et Pierre Nora le rappelle avec insistance) est trace, question et tri. L'histoire tend, avec des modalités et des intensités diverses liées elles-mêmes à des moments divers, à faire reculer, à réduire la mémoire pour s'y substituer et la supplanter:

"Mémoire, histoire : loin d'être synonymes, nous prenons conscience que tout les oppose. La mémoire est la vie, toujours portée par des groupes vivants et à ce titre, elle est en évolution permanente, ouverte à la dialectique du souvenir et de l'amnésie, inconsciente de ses déformations successives, vulnérable à toutes les utilisations et manipulations, susceptible de longues latences et de soudaines revitalisations. L'histoire est la reconstruction toujours problématique et incomplète de ce qui 
n'est plus. (...) Au cœur de l'histoire, travaille un criticisme destructeur de mémoire spontanée. La mémoire est toujours suspecte à l'histoire dont la mission vraie est de la détruire et de la refouler. L'histoire est délégitimation du passé vécu » (Nora 19-20).

Ce que montre aussi Pierre Nora à propos de ce couple mémoire/histoire, c'est que la France a été un pays où l'histoire de la nation a été imposée de manière longtemps efficace comme mémoire collective ou plus précisément comme tradition de mémoire nationale. Avec des approfondissements intellectuels successifs et à travers une pédagogie dont on sait le rôle éminent qu'elle joua dans une cohésion nationale et patriotique que les ébranlements sociaux, politiques, religieux et culturels de la Révolution française à la défaite de 1870 rendaient nécessaire. L'histoire de la nation française fut alors conçue comme le capital mémoriel essentiel. Capital issu de l'accumulation de l'histoire positiviste. Pierre Nora écrit à propos de la grande synthèse opérée par la Troisième République et qui se voulait héritière (au prix de multiples réévaluations, réajustements et réécritures) d'un legs continu allant des chroniqueurs du moyen âge à Augustin Thierry et Michelet pour aboutir au manuel scolaire de Lavisse :

« Histoire, mémoire ont entretenu alors plus qu'une circulation naturelle : une circularité complémentaire, une symbiose à tous les niveaux, scientifique et pédagogique, théorique et pratique. La définition nationale du présent appelait alors impérieusement sa justification par l'éclairage du passé » (Nora 21-22).

Pierre Nora écrit encore à propos de cette conjonction durable mais promise à se défaire sous le coup de réorientations et de poussées désacralisantes successives :

« Dans la perspective finalisée d'une constitution nationale, le politique, le militaire, le biographique et le diplomatique sont (...) les piliers de la continuité. La défaite d'Azincourt ou le poignard de Ravaillac, la journée des Dupes ou telle clause additionnelle des traités de Westphalie relèvent d'une comptabilité scrupuleuse. L'érudition la plus pointue ajoute ou retranche un détail au capital de la nation. Unité puissante de cet espace mémoriel : de notre berceau gréco-romain à l'empire colonial de la Troisième République, pas plus de césure qu'entre la haute érudition qui annexe au patrimoine de nouvelles conquêtes et le manuel scolaire qui en impose la vulgate. Histoire sainte parce que nation sainte. C'est par la nation que notre mémoire s'est maintenue sur le sacré » (Nora 22). 
Cette histoire nationale enseignée massivement et par là même appelée à constituer pour un temps une mémoire nationale soudant les Français sur les valeurs considérées comme essentielles pour la nation et pour l'humanité tout entière a commencé à se défaire au lendemain de la Première Guerre mondiale (l'on pense à certaines élites intellectuelles, littéraires et artistiques telles que les surréalistes) et peut-être plus encore dans les années 1960, contrecoup notamment des guerres de décolonisation en Indochine et en Algérie. L'histoire en France a tendu alors à se délivrer de l'identité nationale pour tenter de devenir tout d'abord science sociale, savoir de la société sur elle-même. Alors que la politique étatique de la mémoire tentait, non sans soulever de nombreuses et véhémentes controverses, de demeurer rivée à ses prétendues certitudes. L'on pense bien évidemment à la loi du 23 février 2005, abrogée un an plus tard, loi invitant à faire en sorte que " les programmes scolaires reconnaissent le rôle positif de la présence française (c'est-à-dire coloniale) outre-mer, notamment en Afrique du Nord et accordent à I'histoire et aux sacrifices des combattants de l'armée françaises issus de ces territoires la place éminente à laquelle ils ont droit » (décret 2006-160 du 15 février 2006). Décret qui a suscité un refus argumenté et efficace (reproche de tentative d'instrumentalisation de la mémoire) d'un très grand nombre d'historiens.

Pierre Nora montre également comment la fin de l'histoire-mémoire, l'essor de l'histoire science sociale se manifestent par un souci sans précédent du vestige et de la trace. La société tout entière est atteinte d'une sorte d'obsession de l'archive, liée sans nul doute au sentiment d'un évanouissement rapide et irréversible, d'un effacement quasi immédiat du souvenir. Aucune société dans l'histoire n'a secrété une telle masse d'archives. II y a sans nul doute une volonté générale d'enregistrement que les comportements individuels confirment : en France les recherches généalogiques se multiplient, la notion de patrimoine s'étend considérablement avec la notion de patrimoine immatériel :

"L'archive n'est plus le reliquat plus ou moins intentionnel d'une mémoire vécue, mais la sécrétion volontaire et organisée d'une mémoire perdue. (...) Le devoir de mémoire fait de chacun l'historien de soi » (Nora 28).

Mais il faut également prendre en compte le développement sans précédent d'un souci épistémologique au sein même des sciences humaines et notamment de la discipline historique.

Est intervenu en outre le fait que depuis la fin du XIXe siècle, depuis la destruction des grands équilibres traditionnels, aussi bien avec Bergson qu'avec Proust et Freud, la mémoire est apparue au centre de la réflexion philosophique, psychologique et littéraire. L'individu devient le refuge d'une mémoire vivante éteinte dans la collectivité : 
« Moins la mémoire est vécue collectivement, plus elle a besoin d'hommes particuliers qui se font eux-mêmes des hommes mémoires " (Nora 30).

De plus les travaux historiques les plus marquants des cinquante dernières années semblent animés par un souci de saisir l'altérité, de souligner l'écart et la différence. Ces historiens n'ont pas tant cherché les grandes continuités que les fractures, les ruptures et les marges. Enfin est venu le temps des lieux de mémoire. Il s'agit tout à la fois d'espaces et d'objets matériellement circonscrits mais surtout marqués d'une forte charge symbolique et impliquant divers rituels : la visite, le recueillement, un type de déambulation de recours ou de consultation s'il s'agit d'un objet ou d'un livre. Il s'agit d'espaces et d'objets suscitant et cristallisant une volonté de mémoire. Ce ne sont pas de simples lieux d'histoire. Par-delà la distinction entre lieux monumentaux et commémoratifs, lieux architecturaux et objets marquants d'une mémoire nationale, Pierre Nora insiste sur l'antithèse des lieux de mémoire dominants et des lieux de mémoire dominés :

« On opposera les lieux dominants et les lieux dominés. Les premiers, spectaculaires et triomphants, imposants et généralement imposés, qu'ils le soient par une autorité nationale ou par un corps constitué, mais toujours d'en haut, ont souvent la froideur ou la solennité des cérémonies officielles. (...) Les seconds sont les lieux refuges, le sanctuaire des fidélités spontanées et des pèlerinages du silence " (Nora 40).

Cette antithèse nous la retrouvons avec des dimensions toutes particulières et aussi avec des inversions dans les pays ou les régions qui ont connu de brutales ruptures politiques ou des évolutions tangibles du même ordre. Nous pensons bien sûr tout d'abord à l'espace même où se tient ce colloque - à ce que furent successivement le royaume du Monténégro, le Royaume des Slovènes, des Serbes et des Croates, le Royaume de Yougoslavie, la Yougoslavie occupée et divisée mais aussi résistante et antifasciste de la seconde guerre mondiale, la République fédérale et socialiste du Maréchal Tito. Nous pensons à l'Espagne libérée du franquisme en 1975, aux espaces mémoriels de la dictature (la Valle de los Caidos) et aux espaces incernables des victimes de la dictature - innombrables fosses communes de la guerre civile, tombe anonyme et décidément introuvable du poète fusillé Federico Garcia Lorca). Que dire du Maroc dont parleront mieux que moi nos collègues de l'Université Moulay Ismail de Meknès contributeurs de ce colloque consacré à la question de la mémoire ? Là aussi nous retrouvons la grande dichotomie des espaces mémoriels dominants et des espaces dominés, dont les dénominations furent occultées voire interdites, durant ce que l'on nomme "les années de plomb » - le long règne autoritaire 
et répressif de Hassan II ? Et je pense bien sûr d'abord au terrible bagne de Tazmamart qui, bien que matériellement effacé, hante la production littéraire contemporaine. Dans chaque espace national concerné, avec sa configuration présente identique ou transformée, il s'agit en fait de tenter d'analyser et de comprendre non seulement le réseau articulé et explicite de la mémoire mais l'organisation inconsciente de la mémoire collective. Ce qui nous permettra peut-être de mieux appréhender des phénomènes actuels globaux très divers tels que la renaissance du roman historique, le succès du récit d'histoire orale et cet attachement pour les lieux où se cristallise et se condense ultime refuge (je reprends une fois encore les mots de Pierre Nora), " le capital épuisé de notre mémoire $"$.

Venons-en maintenant à la perspective difficile de l'étude croisée des mémoires de nos deux espaces : la France et les entités nationales plurielles issues de l'ancienne Yougoslavie. A première vue, en ce qui concerne les politiques de la mémoire, envisager la mise en regard de la France de tradition centraliste par-delà ses révolutions et des Etats issus de l'éclatement de la Yougoslavie semble ne pouvoir aboutir qu'à un constat dıune antinomie radicale. II ne faut cependant pas perdre de vue le fait que la constance et le caractère cohérent et unitaire de la politique française de la mémoire ont pu et peuvent encore apparaître comme un trompe l'œil. Ces quarante dernières années, les travaux des historiens ont en quelque sorte sapé un unanimisme mémoriel français hérité tout à la fois de la Troisième République et, pour la mémoire d'événements plus récents, d'un certain consensus mémoriel gaulliste-communiste postérieur à 1945. Les recherches novatrices des historiens de la Révolution française, antérieurs et contemporains de la grande commémoration du bicentenaire de 1989 (je pense notamment aux travaux de François Furet, Mona Ozouf et Bronislav Baczco) ont conduit à amplifier la part proprement scientifique de la commémoration, à multiplier les débats contradictoires. Les travaux des historiens de la colonisation et de l'esclavage ont pour leur part contraint à renoncer à un discours traditionnel valorisant une colonisation intégralement civilisatrice et partie prétendument incessible du « récit national » (expression très récurrente ces dernières années). Autres occultations mémorielles plus récentes mises en cause par les recherches d'historiens : le rôle de la France de Vichy, de Pétain et de son équipe, de la collaboration française avec le nazisme dans l'organisation du génocide juif, rôle initialement mis à jour par l'historien américain Paxton et finalement officiellement reconnu par le Président de la République Jacques Chirac dans son discours de commémoration de la rafle du Vel d'Hiv en 1995.

C'est dire que dans la France actuelle profondément marquée par une recherche historique amplement diffusée, la mémoire collective s'avère instable, suscite des débats contradictoires parfois virulents et s'avère susceptible 
d'infléchir la politique de la mémoire conçue par les diverses instances d'Etat. La mémoire collective de la France contemporaine soit donc être appréhendée dans ses questionnements, dans ses tensions et dans son instabilité actuelle. Elle ne peut plus être appréhendée dans l'unité qui fut la sienne sur la longue période qui va de la création de l'école laïque (1880) à l'effondrement de 1940, ni dans le consensus des lendemains immédiats de la seconde guerre mondiale. II n'est plus en France de mémoire nationale pleine et entière. Cette mémoire saisie et ébranlée par l'histoire est tissée de tensions.

Pour ce qui concerne le Monténégro dans les années qui ont précédé et suivi l'indépendance, plusieurs constats s'imposent. La première évidence est la résurgence flagrante d'une mémoire pour l'essentiel royale et dynastique dominée par les figures du roi Nicolas, dont le retour des cendres marqua un temps fort de la marche vers l'indépendance, et à l'arrière-plan la prestigieuse figure royale mais aussi poétique et philosophique de Njegoš. Figure qui n'avait jamais été occultée comme en témoigne le deuxième état de son mausolée mais à laquelle on restitua sa dimension de monarque éclairé. A l'heure où Titograd reprenait sa dénomination ancienne de Podgorica, cette résurgence de la mémoire monarchique se manifestait avant tout dans sa dimension monumentale (statues équestres du roi Nicolas avec ce paradoxe de bronzes monumentaux dépourvus de patine). Mais au Monténégro, et cela semble se vérifier dans les temps les plus récents, cette résurgence $n$ 'impliquait en rien l'effacement des références mémorielles à la résistance antifasciste. Ce qui est loin d'être le cas, semble-t-il, dans d'autres républiques issues de l'éclatement de ce qui fut la Yougoslavie. Par ailleurs demeuraient et demeurent de nombreuses traces de l'époque socialiste : monuments, sections de musée consacrées à la résistance antifasciste. Mais au-delà des traces de l'époque du Maréchal Tito, que de traces arasées pour de simples raisons urbanistiques ou économiques. Reste enfin la question des noms de lieux, rues, places, avenues. Dans chacune des républiques indépendantes ces données méritent d'être relevées, rattachées aux dynamiques politiques, culturelles et mémorielles qui les sous-tendent. Une question globale mérite d'être posée pour l'ensemble de la région : jusqu'où est-on allé, jusqu'où va-t-on en matière de mémoire ici ou là ? Qu'est-ce qui a été effacé ? Quelles ont été les résurgences ? Peut-on les dater et les situer exactement ? Ce sont là autant de questions que je me pose à Podgorica comme à Zagreb, à Banja Luka comme à Sarajevo, à Ljubljana comme à Skopje.

Plusieurs publications en langue française permettent de mieux appréhender cette question de la mémoire. L'étude de Valérie Rosoux, Pièges et ressources de la mémoire dans les Balkans publiée à l'Université catholique de Louvain (Belgique) en novembre 2002 permet de mieux saisir ce que furent ces trente dernières années (et ce que sont sans doute encore) les instrumentalisations de la mémoire dans l'aire de l'ex-Yougoslavie. Même si dans l'enchevê- 
trement des conflits des années 1990 référence fut souvent faite à des études historiques précises, ce ne furent en fait que des représentations mentales et des mises en scène d'un passé souvent lointain et de leurs protagonistes qui entrèrent en jeu comme autant d'arguments justifiant ou préparant l'acceptabilité de stratégies et d'actes entrepris ou projetés. Quel passé, à des fins identitaires, chaque force en présence a-t-elle fait resurgir et aux prix de quelles reconstructions, de quelles réécritures ? Par-delà les conflits, dans chacune des républiques issues de l'ancienne Yougoslavie, quelles sont les sélections mémorielles qui ont été opérées et se sont provisoirement ou durablement établies ? Ces configurations mémorielles sont-elles exclusives, incompatibles ou la simple reconnaissance de leur coexistence est-elle, sinon politiquement, du moins intellectuellement envisageable?

L'un des aspects les plus significatifs de l'analyse de Valérie Rosoux tient au fait qu'elle prenne en compte le jeu dialectique des représentations mémorielles balkaniques avec celles de l'espace français et plus globalement de l'espace européen. Nous connaissons bien ce qu'a été la fortune en France et en Europe de l'image des Balkans poudrière : image communément reçue dans le premier versant du XXe siècle, oubliée de 1945 aux années 1990 pour resurgir en force avec l'éclatement de la Yougoslavie et les affrontements qui suivirent. Quelles images la France et l'Union européenne ont-elles renvoyé et renvoientelles encore des Balkans et de leur passé ? Dans quelle mesure la France s'estelle autorisée et s'autorise-t-elle encore de son image traditionnelle (stabilité républicaine, pays des droits de l'homme) dans ses prises de position politiques et diplomatiques vis-à-vis de la Yougoslavie engagée dans la dynamique de son éclatement?

A propos de ces jeux de miroir mémoriels entre la France et l'espace yougoslave, une étude publiée en langue française est particulièrement féconde : il s'agit de l'article de Stanislas Sretenović intitulé « Le monument à la France de Belgrade. La mémoire de la Grande Guerre au service de l'action politique et diplomatique » (publiée dans la Revue Vingtième siècle. Revue d'histoire 20122013, n 115). Le 11 novembre 1930, alors que le Royaume des Serbes, Croates et Slovènes vient d'être transformé en Yougoslavie, et que le roi Alexandre impose l'unification et la centralisation de l'Etat autour de sa personne, un monument est érigé à Belgrade en reconnaissance à la France. Et cette inauguration se double d'une cérémonie de commémoration de l'armistice signé à Rethondes le 11 novembre 1918. Stanislas Sretenović, qui se réfère aux analyses de Pierre Nora, décèle derrière ces cérémonies un message adressé à la société du nouvel Etat des Slaves du Sud et pose les deux questions suivantes:

« Comment la culture républicaine, jacobine et laïque française pouvaitelle être appropriée par un système monarchique qui devait fédérer diffé- 
rents peuples, Serbes, Croates et Slovènes, aux cultures très éloignées de la culture française ? Peut-on parler d'une diplomatie de la mémoire ? »

Le recours au modèle français d'une république centralisatrice et plus que jamais consensuelle et unificatrice après les sacrifices de la Grande Guerre consentis par tous participait selon lui d'une « diplomatie de la mémoire » ambiguë, complexe et mouvante au gré des stratégies des deux Etats. Stanislas Sretenović montre comment, sur la base d'une acceptation française, le gouvernement royal inventa, dès les années 1920, une tradition en produisant des cérémonies où les références françaises jouaient un rôle important :

" Il s'agissait de promouvoir l'alliance victorieuse de la France et de la Serbie pendant la guerre comme fondement de l'Etat de tous les Slaves du Sud $»$.

La commémoration du 11 novembre 1918, le rappel du refuge que constitua la France pour quatre mille élèves et étudiants après leur départ de leur retraite de Corfou, rappel du rôle de la France dans la fondation du royaume des Serbes, Croates et Slovènes en 1918-1919 constituèrent des thématiques obligées des discours et des points d'appui pour une France attachée à renforcer son rôle de grande puissance en Méditerranée et aux confins d'une Europe centrale mal connue parce que longtemps chasse gardée des Habsbourg. Francophonie et francophilie, comme en Roumanie, constituèrent autant de cartes pour la diplomatie française qui n'avait guère que l'Italie pour rivale.

La donne changea cependant avec l'instauration au début de l'année 1929 de la fin du parlementarisme et le début du gouvernement personnel du roi Alexandre. L'administration locale perdit son autonomie avec la création des banovine, provinces administratives nouvelles. A cette époque, dans le royaume de Yougoslavie, tout (nomenclature des rues, art monumental, architecture,) semblait viser à éterniser le rôle de la France. La construction à Belgrade du monument à la France (l'érection d'un monument dédié à un pays étranger était un fait exceptionnel) fut une initiative privée : elle fut le fait de la Société des amis de la France composée pour l'essentiel d'anciens élèves des écoles françaises et un comité du monument fut créé (Niko Miljani, futur cadre du Parti communiste du Monténégro, en était le président). L'emplacement choisi fut celui du buste, détruit en 1915, de Karađođe, leader de l'indépendance. Le choix du lieu, le choix d'un sculpteur croate) furent fortement influencés par l'ambassade de France. L'ambassadeur de France intervenant dans la presse yougoslave pour attribuer au monument une portée symbolique dans tous ses éléments. La construction de la somptueuse ambassade de France non loin du monument s'inscrivait dans la même logique. Stanislav Sretenović montre de 
plus comment la composition de la délégation française lors de l'inauguration, la mise à l'écart des philoserbes français les plus connus répondait à un souci politique précis : signifier que la France ne cautionnerait pas toute démarche des dirigeants serbes. Stanislav Sretenović montre en outre comment la cérémonie d'inauguration en présence du roi le 11 novembre 1930 répondait également du côté yougoslave à de subtils équilibres. Un autre geste impliquant lui aussi une dimension diplomatique fondés sur la mémoire ne semble pas avoir suscité de commentaire dans la presse des deux pays : l'envoi à Belgrade d'une copie de la statue d'Etienne Marcel, prévôt des marchands qui, au XIV siècle tenta de limiter les pouvoirs du roi Charles V. Tout indique que la France ne soutenait qu'avec une grande prudence l'évolution autoritaire du régime. Du côté de l'Etat yougoslave, la référence à la France visait à conforter l'idée d'un Etat fort et d'une Nation unique. Mais l'essentiel de l'étude de Stanislav Sretenović tient au fait qu'il montre l'instrumentalisation, à diverses époques et dans des contextes politiques divers, de la mémoire du soutien de la France à la Serbie durant la Grande Guerre.

Il est sans nul doute d'autres recherches sur ces dynamiques complexes de la mémoire dans l'aire balkanique et peut-être sur la question plus précise de la mémoire des relations politiques, militaires et plus globalement culturelles des relations de l'aire yougoslave avec la France. Pour le Monténégro, il y a bien sûr les travaux d'Ivona Jovanović, issus de sa quête méthodique et scrupuleuse des traces des médiations les plus diverses qui sont à l'origine d'une francophonie et d'une francophilie dont, depuis plus de vingt années d'intervention dans l'Université du Monténégro, je mesure toute l'importance : de la cour de Cetinje à l'aube du XXe siècle, à l'implantation et au développement de l'enseignement de la langue française.

C'est peut-être plus précisément cette question des liens de la langue et de la mémoire qu'il serait utile d'approfondir : une langue nationale est certes porteuse de mémoire mais l'implantation et le développement de la pratique d'une langue étrangère est elle aussi d'autant plus vectrice de représentations mémorielles que le contexte historique a permis le partage d'expériences communes. Souhaitons que les débats et les échanges que permet ce colloque ouvrent la voie de recherches de ce type.

\section{Références bibliographiques}

Jovanovic, Ivona. Langue et culture françaises au Monténégro (1830-1914). Podgorica : Université du Monténégro, Fondation Petrović Njegoš, 2016. Nora, Pierre. Les Lieux de mémoire. Paris : Gallimard, 1984.

Rosoux, Valérie. Pièges et ressources de la mémoire dans les Balkans. Université catholique de Louvain, 2002. 
Sretenović, Stanislav. « Le monument à la France à Belgrade. » Vingtième Siècle. Revue d'histoire 3 (2012): 31-44.

\section{MEMORY OF THE BALKANS, MEMORIES OF FRANCE(ES): TOWARDS RECO- GNITION OF MULTIPLE AND NON EXCLUSIVES MEMORIES}

The contemporary approach to memorial memory in France is quite different from the one applied in the 1990s in the countries of the former Yugoslavia. Nevertheless, the authors of the article have tried to compare them, relying primarily on the concept represented by Pierre Nora in the work of Les Lieux de mémoire, as well as on the distinction the author makes between the notions of memory and history. A certain tradition of national memory was imposed through the educational system in the Third Republic in France. But, in the early 1960s, the historical researches largely contributed to the differentiation from the traditionalist approach of interpreting history as a national novel: history was increasingly recognized as a social and anthropological discipline and the issues of an epistemological (history of history), theoretical and methodological nature were highlighted accordingly.

The attention of researchers and a wide readership stays occupied by controversy over the interpretation of contemporary events (WWII, decolonization). In the wake of the brutal events of the 1990s, which resulted in the rebirth of different entities from the former Yugoslavia, the main antithesis of the place of memorial memory (which applies only to Yugoslavia / which refers to new, national entities) has, in some ways, been transformed. And the transformation was quite unbalanced, given that the commemoration of memorial events by the newly-created states is, above all, a matter of political choice. Each newly formed state asks its own questions: What has been deleted? What came to light and at what cost? How did each state instrumentalize its historical memory in the specific context?

Keywords: Memorial memory, France, Former Yugoslavia, Balkans, history, nationalism / we 